\title{
Prevalencia mundial de la diabetes mellitus tipo 2 y su relación con el índice de desarrollo humano
}

\author{
Miguel Ángel Mendoza Romo, ${ }^{1}$ Aldanely Padrón Salas, ${ }^{2}$ Patricia Elizabeth \\ Cossío Torres ${ }^{2}$ y Manuel Soria Orozco ${ }^{3}$
}

Forma de citar Mendoza Romo MA, Padrón Salas A, Cossío Torres PE, Soria Orozco M. Prevalencia mundial de la diabetes mellitus tipo II y su relación con el índice de desarrollo humano. Rev Panam Salud Publica. 2017;41:e103. doi: 10.26633/RPSP.2017.103

RESUMEN Objetivo. Evaluar la relación entre la prevalencia de diabetes mellitus tipo 2 (DM2) y el indice de desarrollo humano (IDH) por región del mundo en el período 2010-2015.

Método. Se utilizaron los datos de la Federación Internacional de Diabetes para la prevalencia de DM2 (2010-2015) y el IDH del Programa de las Naciones Unidas para el Desarrollo. Se analizaron correlaciones lineales de Spearman entre el IDH y la prevalencia de DM2 y se hicieron regresiones lineales para estimar la relación entre ambos.

Resultados. Se observó que a menor IDH menores son las prevalencias de DM2, y a mayor IDH, mayores son las prevalencias de DM2. A nivel mundial, el IDH explica 8,6\% de la varianza de la prevalencia de DM2 $(P<0,0001)$ y que este comportamiento fue diferente en cada región del mundo.

Conclusiones. El IDH puede influir en la prevalencia de DM2, aunque la relación depende de cada país, región y año analizado.

Palabras clave Diabetes mellitus tipo 2; índice de desarrollo humano, Programa de las Naciones Unidas para el Desarrollo.

Los grandes cambios sociales y económicos han modificado la morbilidad y mortalidad de los países y explican que ahora afronten el aumento de la prevalencia de enfermedades crónicas como la diabetes mellitus tipo 2 (DM2) (1). La DM2 es una enfermedad crónica multifactorial,

\footnotetext{
1 Instituto Mexicano del Seguro Social, San Luis Potosí, México.

2 Departamento de Salud Pública, Facultad de Medicina, Universidad Autónoma de San Luis Potosí, San Luis Potosí, México. La correspondencia se debe dirigir a Aldanely Padrón. Correo electrónico: aldanely.padron@gmail.com

3 Facultad de Medicina, Universidad Autónoma de San Luis Potosí, San Luis Potosí, México.
}

que discapacita y mata a un gran porcentaje de la población a nivel mundial (2).

La DM2 empobrece a las personas y a sus familias, y a ellas y a los sistemas de salud de los países les impone una enorme carga económica (3). Los gastos totales anuales de esta enfermedad oscilan entre \$US 141,6 millones y 174 mil millones, y se estima que las personas con DM2 gastan al menos el doble de dinero en salud que quienes no la padecen $(4,5)$.

Anteriormente, la DM2 se consideraba una enfermedad de ricos y ancianos (6). En cambio, hoy en día se ha arraigado en los países en desarrollo, puesto que en los últimos años más de $80 \%$ de las muertes causadas por esta enfermedad se han registrado en países de ingresos bajos y medios $y$ se calcula que su carga de morbilidad aumentará en todo el mundo y en particular en países en desarrollo (7-9).

En la actualidad, la prevalencia mundial de la DM2 en personas mayores de 18 años ha aumentado de 4,7\% (108 millones de personas) en 1980 a 8,5\% (422 millones de personas) en 2014 y este aumento ha sido más rápido en los países de ingresos medianos y bajos (8).

$\mathrm{Al}$ investigar las causas del aumento de la prevalencia de la DM2 y de otras enfermedades crónicas como la tuberculosis, la infección por el virus de la 
inmunodeficiencia humana, el asma, la depresión o el cáncer, se ha comprobado que la contribución de los factores biológicos y genéticos no es suficiente para explicarlo (5) y que, en cambio, se han encontrado asociaciones con determinantes sociales, como el nivel socioeconómico, los ingresos, la educación, así como con el índice de desarrollo humano (IDH) (10).

En este sentido, se ha observado que a menor ingreso y educación el riesgo de desarrollar DM2 es de 2 a 4 veces más alto que en las personas con ingresos y educación más altos $(5,11)$. Además, la pobreza se ha asociado con una esperanza de vida más corta y un aumento de la mortalidad, sobre todo la relacionada con enfermedades crónicas como la DM2 (11-14). Por otro lado, diversos estudios sugieren que el nivel educativo es clave para adoptar comportamientos relacionados con la salud, como la nutrición adecuada y la adopción de estilos de vida saludables y, por lo tanto, que es posible que el nivel educativo actúe como causa fundamental de la enfermedad mediante la utilización de recursos como el conocimiento, porque influye en la capacidad de las personas para reducir los riesgos, prevenir o retrasar la aparición de la DM2 (15-20). Por su parte, un IDH bajo se ha asociado con un aumento de la incidencia de mortalidad de enfermedades crónicas, lo que refleja la presencia de desigualdades en los factores de riesgo como el acceso, la calidad, la infraestructura y la cobertura de los servicios de salud (21-23).

Teniendo en cuenta lo anterior, cuando se trata de explicar las condiciones en que viven todos los individuos de un país y cómo éstas se relacionan con la prevalencia de una enfermedad, como la DM2, el IDH, por su composición, se puede considerar como primer indicador a nivel macro de los determinantes sociales de la salud (24).

El IDH se utiliza como una propuesta de aproximación multifacética que permite comparar el desarrollo entre países del mundo. Este índice consta de tres componentes: i) educación, considerado como un importante elemento que ayuda a la población a tener más oportunidades de empleo y desarrollo profesional, ii) salud, representado por la esperanza de vida al nacer, y iii) el producto interior bruto (PIB), representado por el ingreso per cápita (25). Un IDH alto indica que los habitantes de un país tienen una vida larga y saludable, acceso a educación y un estándar de vida satisfactorio, lo cual se reflejaría en un país con baja morbilidad por DM2. No obstante, una mejora de la economía o de los bienes materiales puede no traducirse en valores humanos, buena calidad de vida o bienestar en personas con alguna enfermedad crónica como la diabetes.

Para corroborar lo anterior, el objetivo de este estudio fue evaluar la relación de la prevalencia de la DM2 y el IDH por región del mundo de acuerdo con la información proporcionada por la Federación Internacional de Diabetes (FID) y por país, a fin de conocer su comportamiento a nivel global en el periodo comprendido entre 2010 y 2015.

\section{MATERIALES Y MÉTODOS}

Se realizó un estudio ecológico con información de la prevalencia de la DM2 y del IDH del período comprendido entre 2010 y 2015 de los países de todo el mundo, conglomerados por regiones según la FID (África, América Central y del Sur, América del Norte y el Caribe, Oriente medio y Norte de África, Europa, Pacífico Occidental y Sudeste asiático).

La información de la prevalencia de DM2 se obtuvo de los atlas de la FID publicados para los años 2010, 2011, 2012, 2013 y 2015 (26-30). Para 2014, como no había datos publicados, se calcularon los valores a partir de regresiones lineales de los datos obtenidos en el resto de los años incluidos en el estudio.

Los datos del IDH se obtuvieron a partir de los informes del desarrollo humano del Programa de las Naciones Unidas para el Desarrollo para el intervalo de años comprendido entre 2010 y 2014 y para cada uno de los países participantes (31). Los datos del IDH correspondientes a 2015 se calcularon a través de regresiones lineales utilizando los datos de los 5 años anteriores. Esto se hizo con la finalidad de poder determinar si existe una asociación entre la prevalencia de DM2 y el IDH en el periodo evaluado. Se eliminaron aquellos países que carecían de información suficiente para calcular el IDH o la prevalencia de DM2.

Además, se analizaron las prevalencias de DM2 mayores y menores notificadas entre 2010 y 2015 y se calcularon las medias por país de las prevalencias de DM2 durante el mismo periodo sumando cada una de las prevalencias de DM2 y dividiendo entre 6 . Lo anterior también se calculó para el IDH.

Para realizar el análisis estadístico se analizó correlación lineal entre la prevalencia de DM2 y el IDH con el coeficiente de correlación de Spearman para determinar su asociación y su dirección debido a que al analizar ambas variables las dos tuvieron una distribución no normal $(P<0,001)$. Se construyeron regresiones lineales entre ambas variables por país, región del mundo y continente. Los análisis estadísticos se llevaron a cabo con el paquete estadístico Stata v.12 fijando como nivel de significación estadística una alfa = 0,05 (32).

\section{RESULTADOS}

De un total de 220 países de las siete regiones de la FID, se eliminaron 38 por falta de datos sobre la prevalencia de DM2 o del IDH, y se incluyeron, finalmente, 182 distribuidos de la siguiente manera: Oriente medio y Norte de África 10,4\%, Europa 28,6\%, África 23,6\%, América del Norte y el Caribe 9,3\%, América Central y del Sur 9,9\%, Pacífico Occidental 14,3\%, y Sudeste asiático 3,8\%. (cuadro 1).

La prevalencia de DM2 entre 2010 y 2015 varió entre 0,8\% (Benín, África) y 37,3\% (Micronesia, Pacífico Occidental). Los países que notificaron menor prevalencia de DM2 (entre 0,8 y 2,2\%) durante los seis años analizados fueron Benín, Malí, Ruanda, Mongolia, Islandia, Burundi, Gambia, Uganda, Burkina Faso, Guinea, Guinea Bissau, Níger, Senegal y Sierra Leona, de los cuales $85,7 \%$ pertenecían a África. Los países con mayor prevalencia de DM2 (entre 20,0 y 37,3\%) en el mismo periodo fueron Micronesia, Kiribati, Arabia Saudita, Baréin, Kuwait, Qatar, Vanuatu, Mauricio, Líbano y Palau, de los cuales 50,0\% pertenecían a Oriente Medio y Norte de África.

Según las medias de las prevalencias calculadas por país, aquellos con menor prevalencia fueron Benín, Malí, Gambia, Burundi, Mozambique, Burkina Faso, Guinea-Bissau, Sierra Leona, Uganda y Camboya, de los cuales $90,0 \%$ pertenecían a la región de África. Por otro lado, los países con la media de prevalencia más alta fueron Egipto, Mauricio, Vanuatu, Emiratos árabes Unidos, Baréin, Qatar, Kuwait, Arabia Saudita, Kiribati y Micronesia, 6,0\% de los cuales pertenecían a la región del Oriente Medio y África, y 30,0\%, al sudeste asiático. La región con las medias más elevadas de la prevalencia de DM2 en el período estudiado fue Oriente Medio y Norte de África. Las medias de las prevalencias de los países de esta región fueron diez puntos porcentuales mayores durante los últimos años evaluados y lo opuesto ocurrió en la región de África, donde se 
registraron las menores medias de prevalencia de DM2 (cuadro 2).

El IDH en los últimos seis años varió entre 0,3256 (Níger, África) y 0,9444 (Noruega, Europa). Los países con menor IDH $(0,3256-0,3999)$ en el mismo periodo fueron Níger, Chad, Burkina Faso, República Centroafricana, Eritrea, Sierra Leona, Guinea y Burundi, todos pertenecientes a África, y aquellos con mayor IDH $(0,915-$ 0,9444) entre 2010 y 2015, Noruega, Australia, Suiza, Dinamarca, Países Bajos, Alemania, Irlanda, Canadá, Estados Unidos de América y Singapur, 63,3\% de los cuales pertenecían a Europa.

En cuanto a las medias del IDH por país en el mismo periodo, aquellos con la media más baja (IDH < 0,416) fueron Níger, República Centroafricana, Chad, Eritrea, Burkina Faso, Burundi, Sierra Leona, Guinea, Mozambique y Mali, todos pertenecientes a la región de África. Por su parte, los países con la media más alta fueron Nueva Zelanda, Canadá, Irlanda, Estados Unidos de América, Alemania, Países Bajos, Dinamarca, Suiza, Australia y Noruega, (IDH > $0,91)$. Europa fue la región con mayor IDH y África con el menor (cuadro 2).

Al estimar la prevalencia de DM2 según el IDH se observó que los países con mayor IDH tenían una mayor prevalencia de DM2 y viceversa $(P<0,001)$. En los países con IDH menor de 0,60 la prevalencia de DM2 fue menor de 7 (cuadro 3).

El coeficiente de correlación de Spearman entre el IDH y la prevalencia de DM2 fue de 0,339 $(P<0,001)$ (figura 1$)$. Aunque es una asociación leve-moderada, puede observarse una tendencia según la cual a mayor IDH, más alta es la prevalencia de DM2. En los modelos de regresión se observó que la relación entre la variable epidemiológica y la social fue estadísticamente significativa e inversa en el conjunto de los países y años incluidos $\left(r^{2}=9,11\right.$, constante $=1,9$, coeficiente de regresión $=8,6, P<0,001)$. Al analizar esta asociación por años, esta asociación fue estadísticamente significativa y con el mismo patrón en cada uno de los años estudiados $(P<0,05)$ (cuadro 4$)$.

Teniendo en cuenta las regiones de la FID, se encontró que la asociación entre el IDH y la prevalencia de DM2 fue estadísticamente significativa y con el mismo patrón para las regiones de África, Oriente Medio y Norte de África y en el Sudeste asiático, y que el modelo de regresión con el IDH como variable independiente explica hasta $50,9 \%$ de la varianza de la prevalencia de la DM2 $(P<0,001)$ (cuadro 4).

CUADRO 1. Número de países incluidos en el estudio según la Federación Internacional de Diabetes (FID) entre 2010 y 2015

\begin{tabular}{lcccc}
\hline \multicolumn{1}{c}{ Región de la FID } & $\begin{array}{c}\text { Países pertenecientes } \\
\text { a la FID }\end{array}$ & $\begin{array}{c}\text { Países eliminados } \\
\text { Oriente Medio y Norte de África }\end{array}$ & $\begin{array}{c}\text { Total de países } \\
\text { incluidos }\end{array}$ & $\begin{array}{c}\text { Porcentaje de } \\
\text { inclusión }\end{array}$ \\
\hline Europa & 56 & 2 & 19 & 90,48 \\
África & 49 & 4 & 52 & 92,86 \\
América del Norte y el Caribe & 28 & 6 & 43 & 87,76 \\
América Central y del Sur & 20 & 11 & 17 & 60,71 \\
Pacífico Occidental & 39 & 2 & 18 & 90,00 \\
Sudeste asiático & 7 & 13 & 26 & 66,67 \\
Total & 220 & 0 & 7 & 100 \\
\hline
\end{tabular}

Fuente: elaboración propia a partir de la información obtenida de la referencia 30.

CUADRO 2. Medias de la prevalencia de diabetes mellitus tipo 2 (DM2) y del índice desarrollo humano (IDH) por región de la Federación Internacional de Diabetes de 2010 a 2015

\begin{tabular}{lcccccc}
\hline \multicolumn{1}{c}{ Región } & \multicolumn{7}{c}{ Medias de la prevalencia de DM2 } \\
\hline & $2010^{\mathrm{a}}$ & $2011^{\mathrm{b}}$ & $2012^{\mathrm{c}}$ & $2013^{\mathrm{d}}$ & $2014^{\mathrm{e}}$ & $2015^{\dagger}$ \\
África & 4,2 & 4,8 & 4,7 & 5,3 & 4,6 & 4,2 \\
Europa & 6,6 & 6,2 & 6,1 & 6,0 & 6,6 & 7,0 \\
Sudeste asiático & 8,1 & 8,8 & 8,1 & 7,6 & 8,5 & 10,1 \\
América Central y del Sur & 7,5 & 8,5 & 8,4 & 8,1 & 8,8 & 9,1 \\
Paćfico Occidental & 6,6 & 9,4 & 10,3 & 10,6 & 10,5 & 10,2 \\
América del Norte y el Caribe & 9,6 & 11,5 & 12,1 & 11,3 & 12,0 & 12,1 \\
Oriente Medio y Norte de África & 10,8 & 13,5 & 13,6 & 13,6 & 13,3 & 12,7 \\
A nivel mundial & 6,9 & 7,9 & 8,0 & 8,0 & 8,2 & 8,2 \\
Región & & \multicolumn{7}{c}{ Medias del IDH } & & \\
& $2010^{\mathrm{g}}$ & $2011^{9}$ & $2012^{\mathrm{g}}$ & $2013^{\mathrm{g}}$ & $2014^{9}$ & $2015^{\mathrm{h}}$ \\
África & 0,4823 & 0,4887 & 0,4938 & 0,4975 & 0,5012 & 0,5067 \\
Europa & 0,8137 & 0,8178 & 0,8208 & 0,8231 & 0,8251 & 0,8286 \\
Sudeste asiático & 0,6303 & 0,6384 & 0,6439 & 0,6483 & 0,6530 & 0,6595 \\
América Central y del Sur & 0,7182 & 0,7170 & 0,7211 & 0,7236 & 0,7258 & 0,7298 \\
Pacífico Occidental & 0,7111 & 0,7152 & 0,7185 & 0,7209 & 0,7233 & 0,7184 \\
América del Norte y el Caribe & 0,7378 & 0,7398 & 0,7417 & 0,7423 & 0,7445 & 0,7460 \\
Oriente Medio y Norte de África & 0,6965 & 0,6963 & 0,7015 & 0,7023 & 0,7020 & 0,7049 \\
A nivel mundial & 0,6844 & 0,6886 & 0,6924 & 0,6949 & 0,6972 & 0,6999 \\
\hline
\end{tabular}

Fuente: elaboración propia a partir del análisis de la información de las bases de datos de los países seleccionados (referencias 26-30).

a Federación Internacional de Diabetes. Diabetes atlas de la FID. 4ta ed. FID 2010. ํ Federación Internacional de Diabetes.

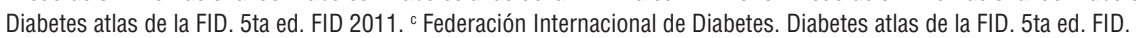

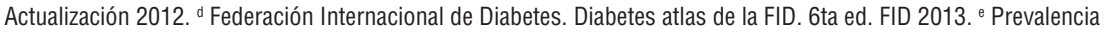
calculada a partir de los años 2010, 2011, 2012, 2012, 2015. ${ }^{\dagger}$ Federación Internacional de Diabetes. Diabetes atlas de la FID. 7 ma ed. FID 2015. (referencias 26-30).

gReferencia 31.

${ }^{\mathrm{h}}$ Datos calculados a partir de los 5 años anteriores.

\section{DISCUSIÓN}

Los estudios ecológicos tienen limitaciones que pueden afectar y explicar los resultados obtenidos. Uno de los principales problemas está relacionado con la calidad de los datos, ya que los sistemas de información en salud de los diferentes países son muy heterogéneos como consecuencia de problemas relacionados con su registro asistemático, la ausencia de soporte magnético, la carencia de formalización institucional de las bases de datos institucional o superposiciones e incoherencias entre bases de datos semejantes, que podrían ocasionar un sobre o subregistro tanto de la prevalencia de DM2 como de la mortalidad utilizada para calcular el IDH (33). Otro problema está relacionado con las pocas actividades de tamizaje y diagnóstico de la DM2 realizadas en diversos países, lo que podría desembocar en una subestimación de su prevalencia (34). Por último, en este 
CUADRO 3. Medias de la prevalencia de diabetes mellitus tipo 2 (DM2) según el índice de desarrollo humano (IDH)

\begin{tabular}{lcccc}
\hline \multirow{2}{*}{ IDH } & $\mathrm{n}$ & \multicolumn{3}{c}{ Prevalencia de DM2 } \\
\cline { 3 - 5 } & & Media & Mínima & Máxima \\
\hline $0,3000-0,3999$ & 37 & 3,9 & 1,8 & 6,3 \\
$0,4000-0,4999$ & 140 & 4,4 & 0,8 & 9,9 \\
$0,5000-0,5999$ & 133 & 6,6 & 2,3 & 27,8 \\
$0,6000-0,6999$ & 157 & 8,5 & 1,6 & 37,3 \\
$0,7000-0,7999$ & 332 & 9,7 & 2,5 & 22,3 \\
$0,8000-0,8999$ & 201 & 8,9 & 1,6 & 23,9 \\
$0,9000-0,9999$ & 92 & 6,7 & 3,2 & 10,7 \\
$P$ & & & $<0,001$ & \\
\hline
\end{tabular}

Fuente: elaboración propia a partir del análisis de la información de las bases de datos de los países seleccionados (referencias 26-31).

FIGURA 1. Correlación entre la prevalencia de diabetes mellitus tipo 2 (DM2) y el índice de desarrollo humano (IDH)

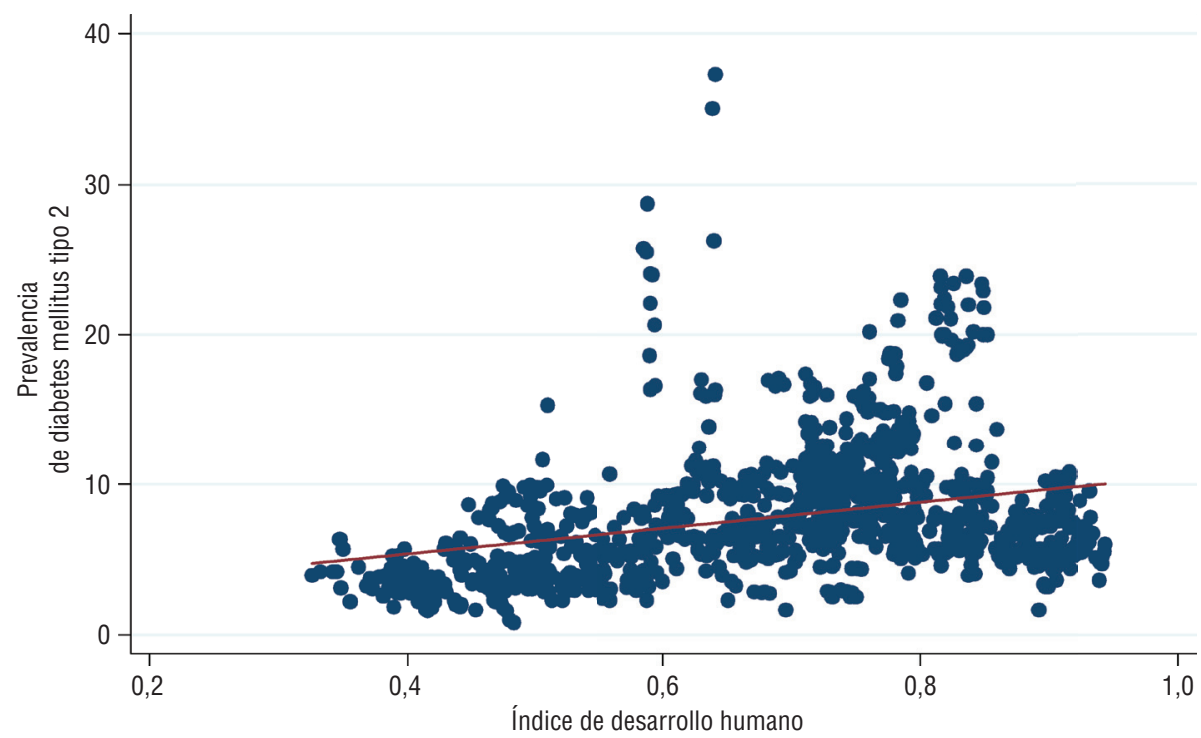

Fuente: elaboración propia a partir del análisis de la información de las bases de datos de los países seleccionados (referencias 26-31).

CUADRO 4. Regresiones lineales para la prevalencia de DM2 según la variación del índice de desarrollo humano por año y región de la FID entre 2010 y 2015

\begin{tabular}{lcccc}
\hline & Constante & Coeficiente & $\mathrm{R}^{2}$ & $\mathrm{P}$ \\
\hline 2010 & 0,5 & 9,4 & 0,224 & $<0,001$ \\
2011 & 2,3 & 8,2 & 0,092 & $<0,001$ \\
2012 & 2,0 & 8,7 & 0,075 & $<0,001$ \\
2013 & 3,9 & 6,0 & 0,036 & 0,0106 \\
2014 & 2,2 & 8,6 & 0,086 & $<0,001$ \\
2015 & 1,1 & 10,1 & 0,126 & $<0,001$ \\
$2010-2015$ & 1,9 & 8,6 & 0,0911 & $<0,001$ \\
África & $-3,1$ & 15,5 & 0,3734 & $<0,0001$ \\
América Central y del Sur & 12,3 & $-5,5$ & 0,0362 & 0,0487 \\
América del Norte y el Caribe & 10,1 & 1,8 & 0,0043 & 0,5134 \\
Europa & 7,8 & $-1,7$ & 0,0050 & 0,2127 \\
Oriente Medio y Norte de África & $-8,4$ & 30,4 & 0,5091 & $<0,0001$ \\
Pacífico Occidental & 11,4 & $-2,50$ & 0,0032 & 0,4851 \\
Sudeste asiático & $-12,0$ & 31,9 & 0,4614 & $<0,0001$ \\
\hline Futerite
\end{tabular}

Fuente: elaboración propia a partir del análisis de la información de las bases de datos de los países seleccionados (referencias 26-31). tipo de estudios se toma como unidad de análisis grupos poblacionales y no al individuo, por lo que la extrapolación de resultados debe hacerse con cautela (33).

No obstante lo anterior, estos estudios son sencillos, fáciles de realizar, de bajo costo y en ellos se utiliza un diseño rápido que permite averiguar la existencia de asociaciones entre fenómenos a nivel poblacional, y en este caso, entre un determinante social y un problema de salud pública mundial. Además, son útiles para formular nuevas hipótesis, sobre todo a partir de la información que se extrae de bases de datos (35).

En el presente estudio se encontró que $9 \%$ de la prevalencia global de DM2 está explicada por el IDH. Sin embargo, hubo regiones, como Oriente Medio y Norte de África, donde este valor alcanzó 50\% y donde se estimaron las medias de prevalencia de DM2 más elevadas. Estos hallazgos indican que un mayor nivel de vida se traduce en mayores prevalencias de DM2. Además, aunque el coeficiente de correlación de Spearman entre el IDH y la prevalencia de DM2 fuese 0,339 y estadísticamente significativo, la correlación es sólo leve-moderada.

Los resultados antes mencionados son incongruentes con los notificados en otros estudios publicados según los cuales a mayor IDH, menor inactividad física en países de bajos y medianos ingresos, porque la inactividad física es un factor de riesgo de la aparición de DM2 (36). Sin embargo, existen otros factores como el estrés y la dieta hipercalórica que desencadenan problemas como la obesidad, el principal factor de riesgo para desarrollar DM2 $(37,38)$.

Por otro lado, en otros estudios donde se buscó una asociación entre el IDH y la prevalencia de depresión (39) y tuberculosis (10) se comprobó que en los países con menor IDH la prevalencia de estos padecimientos fue la más alta, unos resultados que son opuestos a lo observado en la presente investigación probablemente porque pertenecer a un nivel socioeconómico alto implica otros factores relacionados con el estrés al que se somete una persona a diario, como la presión que ejercen cierto tipos de trabajo. En cambio, cuando se intentó asociar el IDH con problemas de salud mental, no se encontró una tendencia lineal, lo que confirma un comportamiento errático de las asociaciones con el IDH (36).

En conclusión, a pesar de que el IDH incluye indicadores de salud, educación y el PIB, la relación de este indicador con la prevalencia de DM2 no muestra una 
tendencia lineal clara debido a que la DM2 es una enfermedad multifactorial y a que, además, podrían influir factores propios del diseño del estudio. Por lo tanto, se propone que, a partir de la información obtenida, se realicen más investigaciones con otro tipo de diseño, que incluya determinantes sociales y permita conocer cómo se

. Federación Internacional de Diabetes. Informe breve. Diabetes: un problema mundial de salud y desarrollo. Bruselas: FID; 2012. Disponible en: http://www.idf.org/sites/ default/files/attachments/PB_GlobalHealth_ES.pdf Acceso el 2 de febrero de 2016.

2. Asociación Americana de Diabetes. Diagnóstico de clasificación de diabetes mellitus. Diabetes Care. 2011;34(1):S62-S69.

3. American Diabetes Association. Economic costs of diabetes in the US in 2012. Diabetes Care. 2013;36(4):1033-46.

4. Charmaine S, Joycee L, Matthias T, Yuko. Cost-of-illness studies of diabetes mellitus: A systematic review. Diabetes Res Clin Pract. 2014;105(2):151-63.

5. Hill J, Nielsen M, Fox MH. Understanding the Social Factors that Contribute to Diabetes: A means to Informing Health Care and Social Policies for the Chronically III. Perm J. 2013;17(2):67-72.

6. Hernández-Ávila M, Gutiérrez JP, Reynoso-Noverón N. Diabetes mellitus en México: El estado de la epidemia. Salud Publica Mex. 2013;55(2):s129-s136.

7. Organización Mundial de la Salud. Diabetes. Día Mundial de la Salud 2016: Vence a la Diabetes. Ginebra: OMS; 2016. Disponible en: http://www.who.int/diabetes/ es/ Acceso el 18 de febrero de 2016.

8. Organización Mundial de la Salud. Diabetes. Centro de prensa. Ginebra: OMS; 2016. Disponible en: http://www.who.int/mediacentre/factsheets/fs312/es/ Acceso el 2 de marzo de 2016.

9. Organización Mundial de la Salud. 10 datos sobre la diabetes. Ginebra: OMS; 2016. Disponible en: http://www.who.int/features/factfiles/diabetes/es/_Acceso el 16 de marzo de 2016.

10. Castañeda-Hernández D, Tobón-García D, Rodríguez-Morales A. Asociación entre incidencia de tuberculosis e Índice de Desarrollo Humano en 165 países del mundo. Rev Peru Med Exp Salud Publica. 2013;30(4):560-8.

11. Rabi DM, Edwards AL, Southern DA, Svenson LW, Sargious OM, Norton P, et al. Association of socio-economic status with diabetes prevalence and utilization of diabetes care services. BMC Health Services Research. 2006;6:124. doi:10.1186/14726963-6-124.

12. Conolly V, Unwin N, Sherriff P, Bilous R, Kelly W. Diabetes prevalence and socioeconomic status: a population based study showing increased prevalence of type 2 diabetes mellitus in deprived areas. J Epidemiol Comm Health. 2000; 54(3):173-7. generan los mecanismos de protección y de riesgo de la DM2 para poder diseñar estrategias específicas adaptadas a cada región o contexto para mejorar la salud de la población.

Financiación. Este estudio no recibió ningún tipo de financiación.

\section{REFERENCIAS}

13. Krishnan S, Cozier Y, Rosenberg L, Palmer J. Socioeconomic Status and Incidence of Type 2 Diabetes: Results from the Black Women's Health Study. Am J Epidemiol. 2010;171(5):564-70.

14. Goldstein J, Jacoby E, del Aguila R, Lopez A. Poverty is a predictor of non-communicable disease among adults in Peruvian cities. Prev Med. 2005;41(3-4):800-6.

15. Borrell LN, Dallo F, White K. Education and Diabetes in a Racially and Ethnically Diverse Population. Am J Public Health. 2006;96(9):1637-42.

16. Brancati FL, Whelton PK, Kuller LH, Klag MJ. Diabetes mellitus, race, and socioeconomic status. A population-based study. Ann Epidemiol. 1996;6(1):67-73.

17. Link BG, Phelan J Social conditions as fundamental causes of disease. J Health Soc Behav. 1995; (Special No):80-94.

18. Choi AI, Weekley CC, Chen S, Li S, Tamura MK, Norris K, et al. Association of Educational Attainment With Chronic Disease and Mortality: The Kidney Early Evaluation Program (KEEP). Am J Kidney Dis. 2011;58(2):228-34.

19. Schillinger D, Barton LR, Karter AJ, Wang F, Adler N. Does literacy mediate the relationship between education and health outcomes? A study of a low-income population with diabetes. Public Health Rep. 2006;121(3):245-54.

20. Domínguez AE. Desigualdades sociales y diabetes mellitus. Rev Cubana Endocrinol. 2013;24(2):200-13.

21. Patel AR, Prasad SM, Shih YT, Eggener SE. The association of the Human Development Index with Global Kidney Cancer Incidence and Mortality. J Urol. 2012;187(6):1978-83.

22. Li-Xia L, Yi Ch, Chao-Hui Y, You-Ming L, Juan Y. National HIV/AIDS mortality, prevalence, and incidence rates are associated with the Human Development Index. Am J Inf Control. 2014;42(10):1044-8.

23. Hassanipour-Azgomi S, Mohammadian-Hafshejani A, Ghoncheh M, Towhidi $\mathrm{F}$, Jamehsorani S, Salehiniya H. Incidence and mortality of prostate cancer and their relationship with the Human Development Index worldwide. Prostate Int. 2016;4(3):118-24.

24. Organización Mundial de la Salud. Determinantes sociales de la salud. Ginebra: OMS; 2016. Disponible en: http://www. who.int/social_determinants/es/ Acceso el 25 de enero de 2016.

25. Morse S. Stirring the pot. Influence of changes in methodology of the Human Development Index on reporting by the press. Ecol Indic. 2014;45:45-54.
Conflictos de interés. Los autores declararan no tener conflictos de interés.

Declaración. Las opiniones expresadas por los autores son de su exclusiva responsabilidad y no reflejan necesariamente los criterios ni la política de la RPSP/PAJPH o de la OPS.

26. Federación Internacional de Diabetes. Diabetes atlas de la FID. 4a ed. Bruselas: FID; 2010.

27. Federación Internacional de Diabetes. Diabetes atlas de la FID. 5a ed. Bruselas: FID; 2011.

28. Federación Internacional de Diabetes. Diabetes atlas de la FID. 5a ed. Bruselas: FID; 2012.

29. Federación Internacional de Diabetes. Diabetes atlas de la FID. 6a ed. Bruselas: FID; 2013.

30. Federación Internacional de Diabetes. Diabetes atlas de la FID. 7a ed. Bruselas: FID; 2015.

31. United Nations Development Programme. Human Development Statistical Tables. New York: UNDP; 2015. Disponible en: http://hdr.undp.org/es/data_Acceso el 25 de enero de 2016.

32. STATA. Data Analysis and Statistical Software. Disponible en: http://www.stata. com/ Acceso el 2 de marzo de 2015.

33. Palacio-Mejía LS, Hernández-Ávila JE, Villalobos A, Cortés-Ortiz MA, Agudelo-Botero M, Plaza B. Sistemas de información en salud en la región mesoamericana. Salud Publica Mex. 2011;53(3):s368-s374.

34. Alazraqui M, Mota E, Spinelli H. Sistemas de Información en Salud: de sistemas cerrados a la ciudadanía social. Un desafío en la reducción de desigualdades en la gestión local. Cad Saude Publica. 2006;22(12):2693-702.

35. Borja-Aburto VH. Estudios ecológicos. Salud Publica Mex. 2000;42(6):33-8.

36. Atkinson K, Lowe S, Moore S. Human development, occupational structure and physical inactivity among 47 low and middle countries. Prevent Med Rep. 2016;3:40-5.

37. Dávila Torres J, González Izquierdo JD, Barrera Cruz A. Panorama de la obesidad en México. Rev Med Inst Mex Seguro Soc. 2015;53(2):240-9.

38. González Jiménez, E. Obesidad: Análisis etiopatogénico y fisiopatológico. Endocrinol Nutr. 2013;60(1):17-24.

39. Cifuentes M, Sembajwe G, Tak S, Gore R, Kriebel D, Punnett L. The association of major depressive episodes with income inequality and the human development index. Soc Sci Med. 2008;67(4):529-39.

Manuscrito recibdo el 11 de agosto de 2016. Aceptado para publicación, tras revisión el 16 de diciembre de 2016. 
ABSTRACT Objective. To evaluate the relationship between the prevalence of type 2 diabetes mellitus (DM2) and the Human Development Index (HDI), by region of the world in the period 2010-2015.

\section{Global prevalence of type 2 diabetes mellitus and its relationship with the Human Development Index}

Keywords
Method. International Diabetes Federation data were used for DM2 prevalence (2010-2015), together with HDI data (United Nations Development Program). Spearman linear correlations between HDI data and DM2 prevalence were analyzed, and linear regressions were done to estimate the relationship between the two.

Results. It was observed that lower HDI scores corresponded to lower DM2 prevalence rates, and higher HDI scores to higher DM2 prevalence. At the global level, the HDI explains the $8.6 \%$ variance of DM2 prevalence $(P<0.0001)$ and shows that the situation was different in each region of the world.

Conclusions. While HDI score may be associated with DM2 prevalence, the relationship between them differs from region to region and from country to country, and depends on the particular year analyzed.

Diabetes mellitus, type 2; human development; United Nations Development Program.
RESUMO

Prevalência mundial da diabetes mellitus tipo $2 \mathrm{e}$ sua relação com o índice de desenvolvimento humano

Palavras-chave
Objetivo. Avaliar a relação entre a prevalência de diabetes mellitus tipo 2 (DM2) e o índice de desenvolvimento humano (IDH) por região do mundo no período de 2010 a 2015.

Métodos. Foram utilizados dados da Federação Internacional de Diabetes para a prevalência da DM2 (2010-2015) e o IDH do Programa das Nações Unidas para o Desenvolvimento. Foram analisadas as correlações lineares de Spearman entre o IDH e a prevalência de DM2 e feitas regressões lineares para estimar a relação entre ambos. Resultados. Observou-se que quanto mais baixo o IDH, menores são as prevalências de DM2, e quanto mais alto o IDH, maiores são prevalências de DM2. Ao nível mundial, o IDH explica 8,6\% da variança da prevalência de DM2 $(\mathrm{P}<0,0001)$ e este comportamento difere em cada região do mundo.

Conclusões. O IDH pode influir na prevalência de DM2, embora a relação dependa de cada país, região e ano analisados.

Diabetes mellitus tipo 2; desenvolvimento humano; Programa das Nações Unidas para o Desenvolvimento. 